\title{
Fast-Gated Single-Photon Avalanche Diode for Wide Dynamic Range Near Infrared Spectroscopy
}

\author{
Alberto Dalla Mora, Alberto Tosi, Member, IEEE, Franco Zappa, Senior Member, IEEE, \\ Sergio Cova, Life Fellow, IEEE, Davide Contini, Antonio Pifferi, Lorenzo Spinelli, \\ Alessandro Torricelli, and Rinaldo Cubeddu
}

\begin{abstract}
We present a novel technique for wide dynamic range optical investigations. It is based on a fast-gated silicon singlephoton avalanche diode (SPAD) in time-correlated single-photon counting (TCSPC) setup. The SPAD is gated-ON and OFF in $500 \mathrm{ps}$ so as to detect photons only within a given time interval. This technique is particularly useful in applications where a large amount of unnecessary photons precede or follow the optical signal to be detected, such as in time-resolved near infrared (NIR) spectroscopy, optical mammography, and optical molecular imaging. In particular, in time-resolved reflectance spectroscopy, it is desirable to minimize the source-detector separation to improve system performance. This leads to the saturation of the detection electronics because of the huge amount of "early" photons back scattered by superficial layers. Our setup is able to reject these photons and detect only "late" photons from the sample, thus allowing an increase in the dynamic range and the injected power. We acquired diffusive curves of two phantoms with 95 ps time resolution and $10^{7}$ dynamic range with a measurement time three orders of magnitude shorter than what is currently possible with a standard TCPSC setup.
\end{abstract}

Index Terms-Avalanche breakdown, infrared spectroscopy, time domain measurements.

Manuscript received July 31, 2009; revised September 14, 2009 and October 12, 2009; accepted October 24, 2009. Date of publication December 8, 2009; date of current version August 6, 2010. This work was supported by the European Community's Seventh Framework Programme FP7 2007-2013 under Grant HEALTH-F5-2008-201076.

A. Dalla Mora and A. Tosi are with the Dipartimento di Elettronica e Informazione, Politecnico di Milano, 20133 Milan, Italy (e-mail: alberto. dallamora@mail.polimi.it; alberto.tosi@polimi.it).

F. Zappa is with the Dipartimento di Elettronica e Informazione, Politecnico di Milano, 20133 Milan, Italy, and also with the Micro Photon Devices S. r. 1., 39100 Bolzano, Italy (e-mail: franco.zappa@ polimi.it).

S. Cova is with the Dipartimento di Elettronica e Informazione and IIT Research Unit, Politecnico di Milano, 20133 Milan, Italy, and also with the Micro Photon Devices S. r. 1., 39100 Bolzano, Italy (e-mail: cova@elet.polimi.it).

D. Contini is with the Dipartimento di Fisica and National Laboratory for Ultrafast and Ultraintense Optical Science - CNR, Politecnico di Milano, 20133 Milan, Italy (e-mail: davide.contini@polimi.it).

A. Pifferi is with the Dipartimento di Fisica and IIT Research Unit, Politecnico di Milano, 20133 Milano, Italy, and also with the National Laboratory for Ultrafast and Ultraintense Optical Science - CNR, Politecnico di Milano, 20133 Milan, Italy (e-mail: antonio.pifferi@ polimi.it).

L. Spinelli is with the Dipartimento di Fisica, IFN-CNR Politecnico di Milano, 20133 Milan, Italy (e-mail: lorenzo.spinelli@ fisi.polimi.it).

A. Torricelli is with the Dipartimento di Fisica and IIT Research Unit, Politecnico di Milano, 20133 Milan, Italy (e-mail: alessandro.torricelli@polimi.it).

R. Cubeddu is with the Dipartimento di Fisica, IIT Research Unit, IFNCNR, and the National Laboratory for Ultrafast and Ultraintense Optical Science - CNR, Politecnico di Milano, 20133 Milan, Italy (e-mail: rinaldo. cubeddu@polimi.it).

Color versions of one or more of the figures in this paper are available online at http://ieeexplore.ieee.org.

Digital Object Identifier 10.1109/JSTQE.2009.2035823

\section{INTRODUCTION}

T IS WELL known and widely discussed in literature that red and near infrared light can be used to noninvasively probe highly scattering media such as biological tissues [1]. In particular, in biomedical optics, photon migration phenomena can be exploited for different and innovative applications, such as functional brain imaging [2], mammography [3], or molecular imaging [4]. In reflectance optical spectroscopy measurements, the diffusive medium is illuminated from a point source and diffused photons are collected at a given distance from the source. Different techniques can be employed to probe media, such as continuous wave, frequency domain, and time domain. Among these, time domain techniques can provide higher sensitivity and penetration depth by exploiting the timing information of scattered photons [5]-[7].

These techniques allow the noninvasive detection in vivo of brain cortex activation by monitoring cerebral hemodynamics (e.g., concentration changes of both oxygenated and deoxygenated hemoglobin), which produce localized changes of optical absorption coefficients [8]. The key point of these measurements is the possibility of increasing the penetration depth of investigation, in particular for functional brain imaging, where skin, skull, and cerebrospinal fluid (CSF) heavily mask the brain signal [8]. In time-resolved techniques, it has been demonstrated that the mean penetration depth of photons detected at the same delay after the injection of the light pulse into the medium is independent of the source-detector separation $\rho$, but it increases with the arrival delay of detected photons [6]. Therefore, the separation between the illumination source and the collection point can be reduced, thus increasing the number of collected photons at any time delay and, at the same time, increasing contrast and spatial resolution [9].

Notwithstanding such drastic improvements, the strong presence of the "early photons" (i.e., photons scattered by surface regions) usually severely limits performance at small sourcedetector distance, since their number overcomes by many orders of magnitude that of "late photons" (i.e., photons back diffused by inner regions). The presence of early photons imposes the need to reduce the optical power injected so as to avoid saturation of the detection system. When using time-correlated singlephoton counting (TCSPC) techniques [10], the maximum count rate is limited both by the saturation threshold of the electronics and by the so-called "pile-up" distortion effect. The latter 
problem arises when more than one photon reaches the detector during the same measurement period and only the first one is detected, because of the dead time of both photodetector and electronics.

In order to attain the best performance at small sourcedetector separation, and to reduce measurement time, the optical power injected into the tissue must be kept as high as possible, but below safety limits for in vivo spectroscopy. This will enhance the small number of "late" photons, which carry information about the deeper regions of the tissues under investigation. Because of the need to detect the very faint signal of longer lived photons, a single-photon detector is required. Moreover, the detector must be time-gated in order to reject early photons, by enabling it after a well-defined delay with respect to laser excitation. The transition from OFF state to ON state must be very fast (few hundreds of picoseconds at most) because of the small time separation between unwanted photons and the useful ones [9].

Some available detectors can be operated in fast-gated mode; for example, microchannel plate photomultiplier tubes (PMTs) by modulating the gain, or intensified charge coupled devices (CCD) by modulating the intensifier, or a streak camera by controlling the ramp voltage. However, in all these detectors, the performances are heavily limited by the fact that the photocathode is always impinged by initial photons, thus heavily enhancing background noise and perhaps causing permanent damage.

In [11], we demonstrated for the first time the feasibility and advantages of minimizing the source-detector separation in time-resolved reflectance NIR spectroscopy, by means of a fast-gated silicon single-photon avalanche diode (SPAD) [12], [13]. In this paper, we discuss the proposed technique, and we describe the electronics and the experimental characterization of the overall time-gated SPAD equipment.

The SPAD is a p-n junction-biased above the breakdown voltage $V_{\mathrm{BD}}$, in the Geiger-mode operating regime. At this bias, the electric field is so high that a single charge carrier, originating in the depletion region, can trigger a self-sustaining avalanche process by impact ionization. If the primary carrier is photogenerated, the leading edge of the avalanche pulse marks the arrival time of the detected photon with picosecond resolution. After the avalanche is triggered, current keeps flowing until the bias voltage is lowered near to (or below) the breakdown voltage, where the impact-ionization process is no longer self-sustaining, thus quenching the avalanche. Finally, the bias voltage must be restored in order to be able to detect another photon. All these operations require suitable electronics that must: 1) sense the fast leading-edge of the avalanche current; 2) generate a low time-jitter output pulse well synchronized to the avalanche pulse for timing the photon; 3 ) quench the avalanche by lowering the bias voltage; and 4) restore the detector voltage to the operating level. This circuit is called a "quenching circuit" [13], and its performance heavily impacts on detector performance in terms of noise, timing resolution, and maximum counting rate.

In this paper, we describe the novel measurement setup, improved with respect to that proposed in [11], and report performance in terms of instrument response function (IRF), dynamic

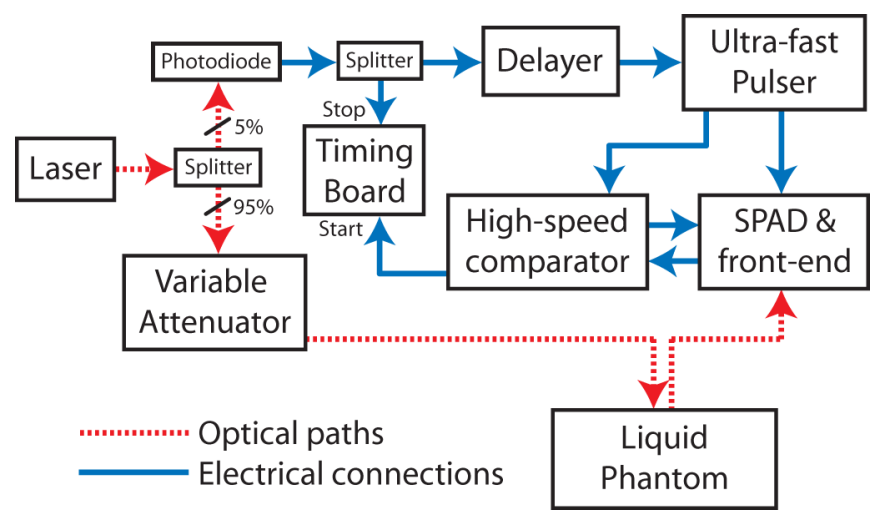

Fig. 1. Complete fast-gated SPAD setup for photon migration measurements.

range and time range. Moreover, we report time-resolved reflectance curves acquired at $2 \mathrm{~mm}$ source-detector separation of two liquid phantoms, each with different absorption coefficients. We prove the effectiveness of the measurement setup for the non-invasive assessment of phantom optical parameters. We obtain a wide dynamic range and strongly reduced the measurement time with respect to traditional free-running operation.

\section{SYSTEM SETUP}

Our setup for fast-gated photon migration measurements is shown in Fig. 1. The optical source is the super-continuum fiber laser SC450 by Fianium Ltd., which provides white-light picosecond pulses at $20 \mathrm{MHz}$ repetition rate. An F2 glass prism (PS854 by Thorlabs Inc.) separates different optical wavelengths, thus allowing the selection of a wide spectral range (between 465 and $1750 \mathrm{~nm}$ ). Then, the light is collected into an optical fiber. A beam splitter (5/95 split ratio) derives the optical pulse to a photodiode, which provides a trigger signal to the following electronics. Then, the light (whose power is regulated by a variable attenuator) is injected by means of $1 \mathrm{~mm}$ core diameter optical fiber into a homogeneous liquid phantom. The scattered pulse is collected by a similar optical fiber, and then it is focused onto the SPAD active area. The silicon SPAD, developed at Politecnico di Milano [14] and employed in commercial modules by micro photon devices [15], has a $100 \mu \mathrm{m}$ active area diameter, a breakdown voltage of about $23 \mathrm{~V}$ and a capacitance between anode and cathode of about $3 \mathrm{pF}$ when biased slightly below the breakdown voltage.

The trigger signal derived from the laser pulse acts as a stop pulse for the time correlated single-photon counting board (SPC130 from Becker and Hickl GmbH), while the start event is provided by the differential high-speed comparator which senses the avalanche pulse of the SPAD. We developed our own fast pulse generator to gate on and off the detector in about 500 ps with low oscillations and disturbance.

A programmable delayer (with minimum time steps of $25 \mathrm{ps}$ ) introduces a well-defined time delay between the trigger signal and the ignition of the fast pulse generator, thus avoiding the detection of early photons. 


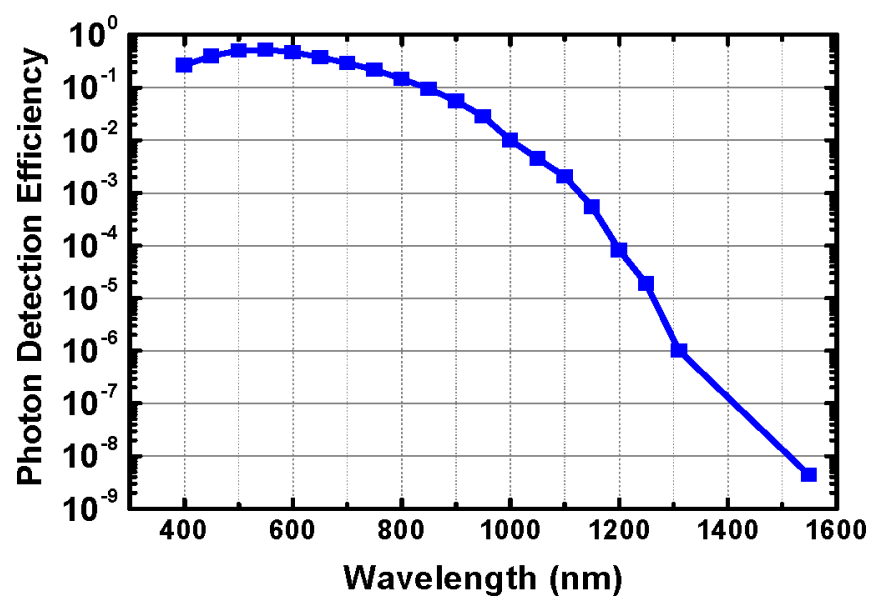

Fig. 2. Photon detection efficiency of the SPAD detector employed in the measurements, operated at an excess bias of $5 \mathrm{~V}$ [16].

\section{SPADS IN FAST GATED-MODE OPERATION}

The exploitation of SPAD detectors in biomedical optical measurements opens new perspectives. Planar silicon SPADs are particularly suited for these kind of applications thanks to: 1) high quantum efficiency (see Fig. 2) allowing the detection of very faint optical signals in a wide spectral range [16];2) low time jitter, few tens of picoseconds in free-running operation; 3) low dark count rate, about $1 \mathrm{kcounts} / \mathrm{s}$ for a device with $100 \mu \mathrm{m}$ active area diameter. Furthermore, with respect to other approaches, planar SPAD detectors can be gated-ON and OFF very quickly, by raising and lowering the bias voltage with respect to the breakdown voltage, which is normally of few tens of volts. When the SPAD is disabled, the primary electronhole pair generated by the incoming photon cannot trigger the avalanche process and recombines in a few tens of picoseconds. In this way, early photons are rejected and thus no detector saturation occurs.

Quenching circuits can generally be either passive or active [13]. In the passive quenching circuit (PQC) approach, the avalanche current flows through a resistor in series with the SPAD, thus developing a voltage drop that swiftly reduces the bias voltage across the detector and, eventually, quenches the avalanche. In order to completely quench the current, the resistor must have a high value (hundreds of $\mathrm{k} \Omega$ ). Conversely, in an active quenching circuit (AQC) [17], fast electronics detects the avalanche ignition and subsequently forces the bias voltage below the breakdown level; afterwards it restores the reverse voltage to the operational level through an active transition. The drawback of this approach is that the gate transition duration is limited to few nanoseconds because of parasitic capacitances of both detector and AQC (mainly in the discretecomponent approach) and limited current driving capabilities (e.g., of CMOS transistors in the integrated-circuit approach). Because of these limitations, the AQC approach is not effective when ultrafast gating is required.

Fig. 3 shows a typical SPAD front-end circuit, useful for gated-mode operation. The negative reverse voltage $V_{\mathrm{REV}}$ is slightly lower than $V_{\mathrm{BD}}$ (e.g., $0.5 \mathrm{~V}$ lower), and it is applied to

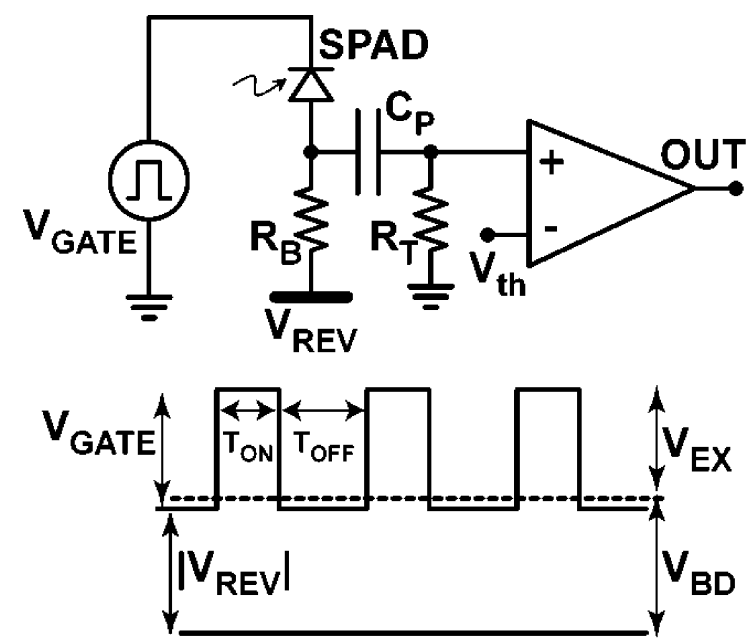

Fig. 3. Passive quenching circuit (top) and SPAD voltage waveform (bottom) for gated-mode operation (when no avalanche occurs): the SPAD is operative only during the $T_{\mathrm{ON}}$ window, when the reverse SPAD voltage exceeds the breakdown voltage $V_{\mathrm{BD}}$ by an amount $V_{\mathrm{EX}}$.

the anode by means of the ballast resistor $R_{\mathrm{B}}$ (few hundreds of $\mathrm{k} \Omega$ ). For instance, with our SPAD with $V_{\mathrm{BD}}=23 \mathrm{~V}$, we employed $V_{\mathrm{REV}}=-22.5 \mathrm{~V}$. During the gate-off time interval $\left(T_{\mathrm{OFF}}\right), V_{\mathrm{GATE}}$ is $0 \mathrm{~V}$, hence the SPAD voltage is lower than $V_{\mathrm{BD}}$ and no avalanche can be triggered. When $V_{\mathrm{GATE}}$ is set to the high level (e.g., $V_{\mathrm{GATE}}=5.5 \mathrm{~V}$ ) during the gate-ON time interval $\left(T_{\mathrm{ON}}\right)$, the SPAD voltage (i.e., $\left.\left|V_{\mathrm{REV}}\right|+V_{\mathrm{GATE}}\right)$ goes beyond $V_{\mathrm{BD}}$ by an amount called excess bias voltage $V_{\mathrm{EX}}$. When an avalanche is triggered, the current pulse is sensed by the ac coupled comparator (whose threshold is $V_{\mathrm{th}}$ ), through the pick-up network composed of $C_{P}$ (tens of $\mathrm{pF}$ ) and $R_{T}$ (tens of $\Omega$ ).

When the avalanche is triggered, the reverse current starts to flow through the photodetector. $R_{B}$ being much higher than $R_{T}$, most of the building-up avalanche current flows through $C_{P}$ and $R_{T}$ : the fast discharging of $C_{P}$ allows the reverse SPAD voltage to lower quickly, thus promptly quenching the avalanche. After quenching, the resistor $R_{B}$ brings back the anode voltage to $V_{\mathrm{REV}}$, thus restoring the initial operating conditions. Unfortunately, such transition involving $C_{P}$ and SPAD capacitance is quite long due to the high $R_{B}$ value, thus heavily limiting the maximum count rate.

A second issue of the circuit in Fig. 3 is the presence of a strong disturbance at the comparator input. In fact, when a fast gate pulse is applied to the cathode, the anode experiences a sudden variation because of the voltage divider between the SPAD capacitance and the pick-up network. This ac coupling gives rise to an intense and fast spurious voltage spike at the comparator input [18]: inevitably, the faster the rise and fall times, the higher the spurious peaks. Fig. 4 shows the signal at the comparator input of Fig. 2, when rise and fall gate transitions of $200 \mathrm{ps}$ (measured from $20 \%$ to $80 \%$ of the voltage swing) and 5-ns pulse duration $\left(T_{\mathrm{ON}}\right)$ are employed. The avalanche pulse is three times weaker than the rising-edge spike, so photons cannot be detected by means of standard gated-mode front-end circuits. 


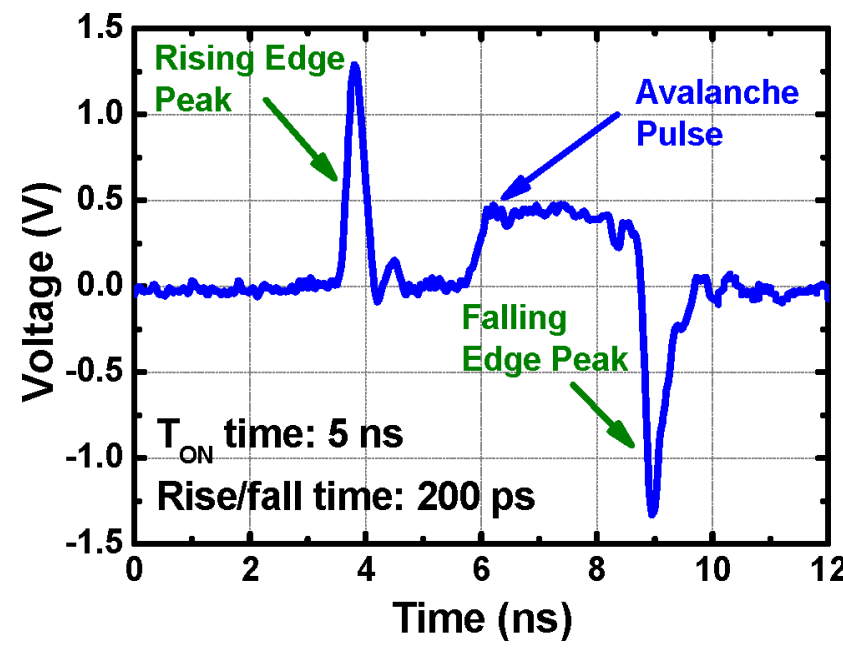

Fig. 4. Signal at the input of the comparator of Fig. 3, with 200 ps rise/fall gate transitions and a gate voltage amplitude of $5.5 \mathrm{~V}$. The spurious risingedge peak is three times higher than the avalanche pulse and always triggers the comparator, thus hiding any photon detection with standard single-ended pick-up circuits.

This problem is well known in the literature, especially for InGaAs SPADs, which require gated-mode operation because of the high-dark count rate and afterpulsing, and some approaches have been proposed. One exploits reflections at the ends of a network of coaxial cables for obtaining the cancellation of the spurious spikes [19]. Another approach [20] cancels spikes by means of a $180^{\circ}$ hybrid junction, whose inputs are the balanced outputs of two identical SPADs. Our solution is to generate an auxiliary "dummy" path that reproduces only the spurious peaks. Then, by means of differential sensing between the SPAD signal and the dummy signal, it is possible to detect the avalanche pulse, hence avoiding any common-mode disturbance [18]. The better the matching between the two paths, the closer the resemblance of the spurious peaks in both signals, the lower the comparator voltage threshold can be, thus enhancing timing resolution [14]. As shown in Fig. 5, the "dummy path" employs a capacitor that mimics the SPAD capacitance in order to generate the dummy signal. Two pick-up capacitors are used to feed the two signals to the high-speed comparator inputs. The ballast resistor has been replaced by a wide-band bipolar transistor that is usually kept off, in order to provide high impedance for the SPAD anode and to guarantee a properly fast quenching transition of the detector. Then, it is turned on at the beginning of the gate-OFF time, in order to "actively" fast restore the initial SPAD reverse voltage by bringing the anode voltage back to $V_{\mathrm{REV}}$. Also the reset network is doubled, for the detector and the dummy path, to provide a better matching of spurious spikes during both gate transitions, thus guaranteeing spike rejection.

A first important feature of the time-resolved measurement setup is the flat response of the detector over the enabling time. Unfortunately, because of both electronics parasitism (e.g., spurious capacitances and inductances) and pulse generator nonidealities (e.g., voltage overshoots and ringing), it is usually hard to end-up with a flat response while gating the SPAD with ultrafast transitions. We measured the time distribution of dark

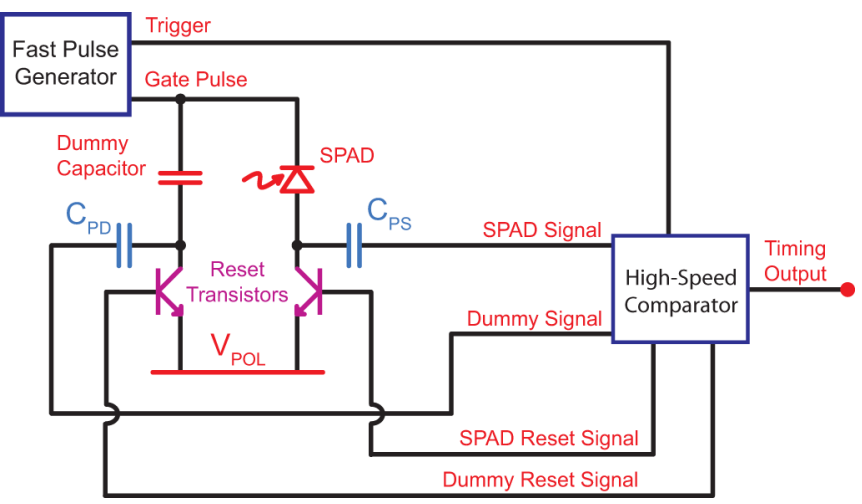

Fig. 5. Fast-gating electronics: the pulse generator provides ultrafast gate pulses to enable the detector, the differential pick-up network allows precise avalanche detection, and the high-speed comparator drives the reset transistors to rearm the SPAD after each ignition.

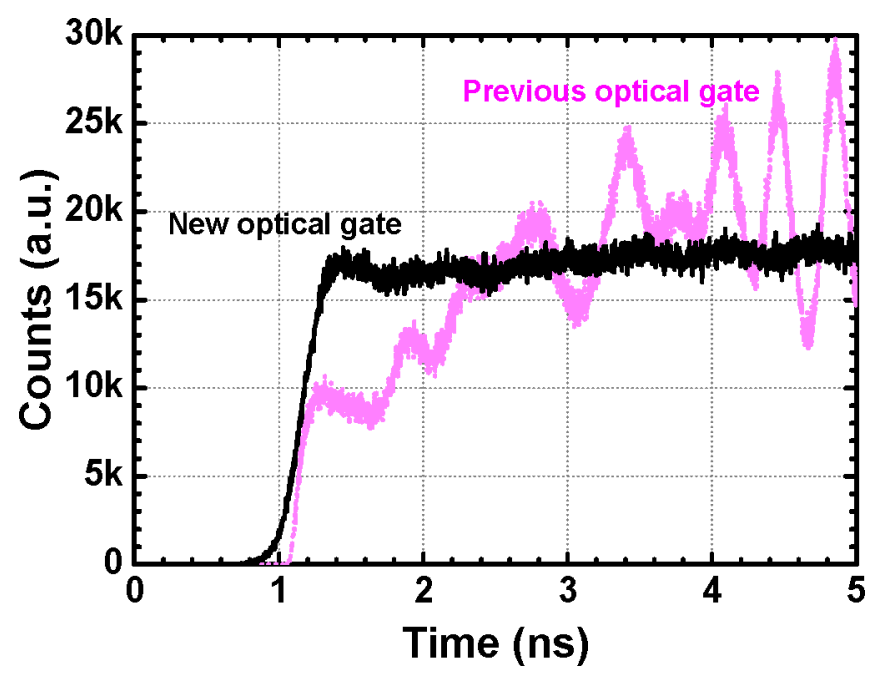

Fig. 6. Flatness of the new SPAD optical response when a fast rising transition (500 ps) is applied to gate-ON the detector kept in the dark (dark counts). Comparison with the previous optical gate of the setup presented in [11].

counts during the gate-ON time to check the timing response flatness of our setup. In this way, since the dark count rate increases almost exponentially with the excess bias [13], any voltage oscillation would be exponentially amplified and would clearly appear as a nonuniform timing response. In Fig. 6, the response obtained with the prototypal solution reported in [11] is reported. As shown, the response has strong voltage oscillations that heavily impacts on photon detection efficiency and detection linearity. Instead, with our custom pulse generator and optimized SPAD front-end electronics, we obtained the flat response shown in Fig. 6 (new optical gate). The measured response is very flat even just after the gate-ON transition. With a 500 ps electrical rising transition applied to the SPAD, the measured optical rise-time (see Fig. 6) is even shorter, lasting about 200 ps (from $20 \%$ to $80 \%$ ), because of the exponential dependence of dark counts on the applied voltage.

A second important detector feature in time-resolved techniques is the time resolution. This property is relevant for separating absorption from scattering effects, in addition the depth 


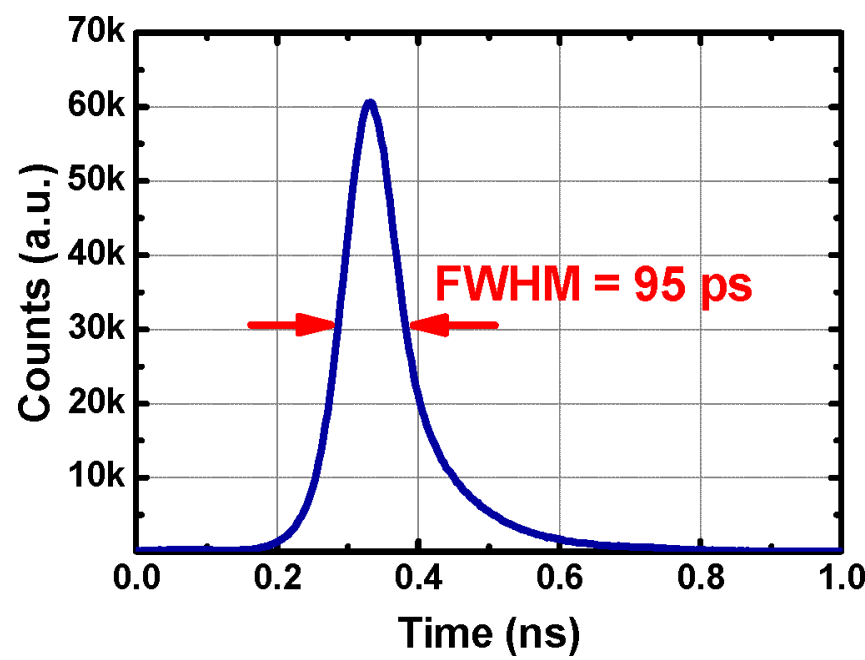

Fig. 7. Timing response of the ultrafast time-gated SPAD. Thanks to the differential pick-up network we measured a time resolution of 95 ps (FWHM) at $5 \mathrm{~V}$ excess bias voltage.

information in reflectance measurements is encoded in photon arrival time. Therefore, a precise temporal resolution of the setup is crucial to pursue optimal depth resolution and sensitivity. In order to measure the time jitter of the fast time-gated SPAD setup, the SPAD was biased just $0.5 \mathrm{~V}$ below the breakdown voltage (see Fig. 3, bottom) and it was gated with ultrafast voltage pulses of $5.5 \mathrm{~V}$ amplitude (the excess bias was $5 \mathrm{~V}$ ), and with a $5 \mathrm{~ns}$ time duration. We selected an $800 \mathrm{~nm}$ optical wavelength at the laser output. The overall instrumental response function time resolution is 95 ps full-width at half-maximum (FWHM), with a remarkable 550 ps full-width at 1/100 maximum (see Fig. 7). Compared with the intrinsic 35 ps FWHM SPAD response time jitter, the broadening to $95 \mathrm{ps}$ is due to the quite high-threshold voltage (about $100 \mathrm{mV}$ ) required to detect only the avalanche pulse and to avoid spurious voltage spikes [14].

\section{SYSTEM CHARACTERIZATION}

In order to assess the wide dynamic range of our setup, we measured the instrument response function (IRF) to a laser excitation pulse and we acquired reflectance curves of two homogeneous phantoms at a minimum source-detector distance of $2 \mathrm{~mm}$ for a wavelength of $800 \mathrm{~nm}$. For the IRF, we directly coupled the excitation optical fiber with the collecting fiber through a diffusing layer of poly(tetrafluoroethylene) (PTFE) in order to guarantee isotropic illumination of the latter. In order to accurately reconstruct the IRF with large dynamic range, we acquired different slices of the optical pulse by fast-gating the detector at different delays with respect to the laser pulse peak. In detail, we started with the optical pulse peak in the middle of the gate-ON time interval, then the gate window was delayed in steps of 25 ps for a total of 201 time intervals with respect to the peak. For each delay, we acquired photons for $10 \mathrm{~s}$, therefore, the total measurement time is $33.5 \mathrm{~min}$. When the laser pulse peak was inside the gate window, the laser power was strongly attenuated (through the variable attenuator shown in Fig. 1) so as to detect at most $0.5 \mathrm{Mcps}$ (counts per second) in order to avoid

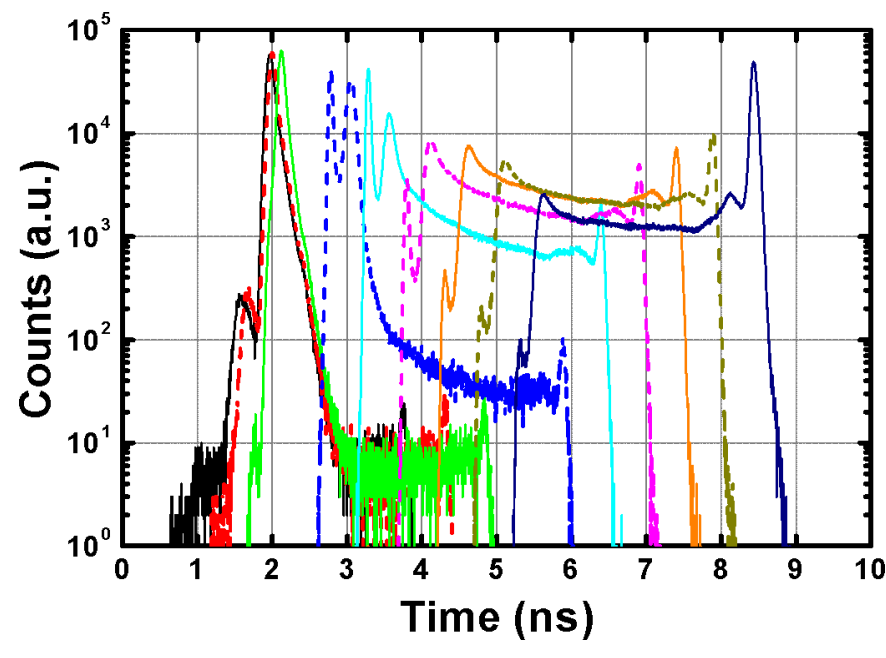

Fig. 8. Some of the 201 acquisitions obtained by delaying the gate-ON time window with respect to the laser pulse excitation.

pile-up distortions during TCSPC measurements [10]. However, when the gating signal was sufficiently delayed, the laser pulse peak was out of the gate-ON window and only the pulse tail was measured. Since the longer the gate delay, the fainter the IRF, we progressively increased the laser power in order to increase the SNR. Fig. 8 shows some of the IRF slices acquired at different delays. In the right-most curves of the plot, it is possible to notice the rise of the tail intensity, due to the increased optical power, which allows an improved SNR in characterization of the last part of the IRF.

By combining slices acquired at the different delays, and taking into account the different attenuation for each delay, it is possible to reconstruct the whole instrument response function shown in Fig. 9. Because of the distortions introduced during the rising and falling edges of the gating pulse, for the reconstruction we discarded the firsts 600 ps of each of the 201 acquisitions, making use of the following $2 \mathrm{~ns}$, where the detector response is very stable. The obtained dynamic range is seven orders of magnitude and allows the detection of photons up to $6 \mathrm{~ns}$ after the laser peak.

Two reflections are present in the optical system (see Fig. 9). The first one (at about $1.5 \mathrm{~ns}$, before the main peak located at $2 \mathrm{~ns}$ ) is due to the previous excitation pulse (about $50 \mathrm{~ns}$ before), and does not impact on the measurement. Instead, the second reflection is within the useful observation time range (at about $8.5 \mathrm{~ns}$ ) and has a detrimental effect. Indeed, when the gate-ON window is delayed to acquire very late photons (at around $6 \mathrm{~ns}$ from the main peak), such reflection shows up and increases when the laser power is further increased (see also the rightmost plots of Fig. 8). Therefore, the laser power cannot be increased as wished, because of saturation of the detection system. Therefore, such reflection limits further extension of both dynamic and time ranges. It is worth noting that, since the amplitude of the second reflection is five orders of magnitude weaker than the main peak, it is very difficult to fully eliminate it. 


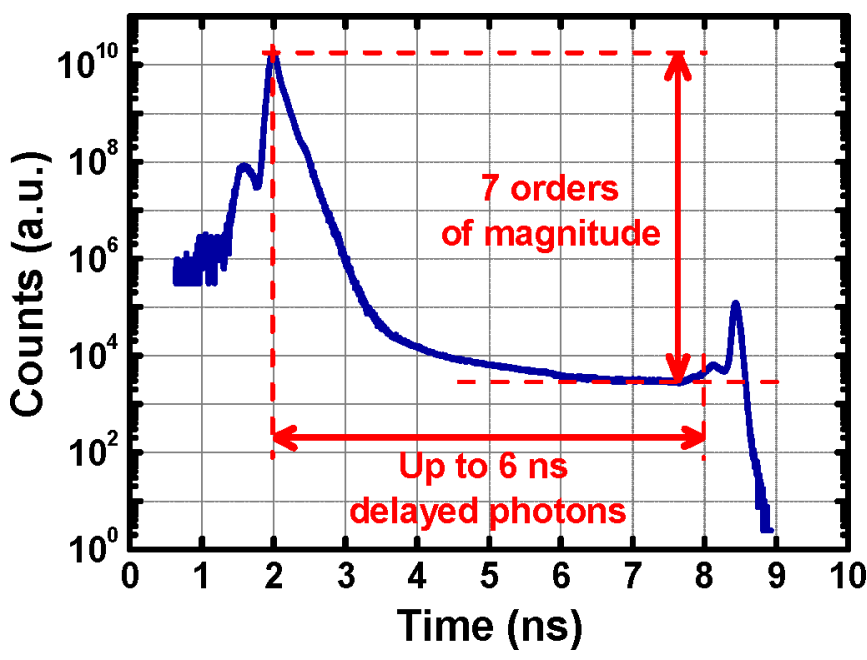

Fig. 9. Reconstructed IRF of the ultrafast-gated SPAD setup. Thanks to the delayed gate measurements it is possible to obtain a wide dynamic range of seven orders of magnitude in a $33.5 \mathrm{~min}$ measurement time.

The reconstructed waveform of Fig. 9 is composed of about $8.69 \times 10^{11}$ counts. It is worth pointing out that this number is not the actual number of collected photons. Each of the 201 acquired slices is multiplied by the reciprocal of the optical attenuation introduced to keep the maximum count rate below 0.5 Mcps. In order to achieve the same dynamic range (and the same number of photons) by means of a traditional TCSPC measurement at the same count rate, about $483 \mathrm{~h}$ would be required. This drastic improvement of roughly three orders of magnitude in measurement time is possible because the SNR is not constant along the reconstructed curve, but it is lower at shorter delays, where the large multiplication factor applied to the acquired counts increases noise as well as signal. However, the overall IRF is still characterized by a fairly low noise, as is clear from Fig. 9.

We also performed measurements of reflectance curves of two homogeneous liquid phantoms [21], with different absorption coefficients (P1 with $\mu_{a}=0.005 \mathrm{~mm}^{-1}$ and P2 with $\mu_{a}=0.01 \mathrm{~mm}^{-1}$ ) and the same reduced scattering coefficient $\left(\mu_{s}^{\prime}=0.5 \mathrm{~mm}^{-1}\right)$, at a $2 \mathrm{~mm}$ source-detector distance. Phantoms are composed of a mixture of distilled water, $20 \%$ intralipid as diffuser, and Indian ink as absorber.

Fig. 10 shows the time-resolved reflectance curves of the phantoms, normalized with respect to the peak value. As a reference, the instrument response function is also reported. We reconstructed curves by employing the same delays and measurement time used for the IRF acquisition and we obtained the same $10^{7}$ wide dynamic range. Phantoms $\mathrm{P} 1$ and $\mathrm{P} 2$ have clearly distinguishable photon time distributions due to the different $a b-$ sorption coefficient. As shown in Fig. 10, such difference can be appreciated from 3 to 7 orders of magnitude below the pulse peak from 0.5 to $4.5 \mathrm{~ns}$ time of flight from the pulse stimulus.

\section{DISCUSSION}

These results demonstrate the possibility of collecting information at long arrival times in time-resolved reflectance NIR

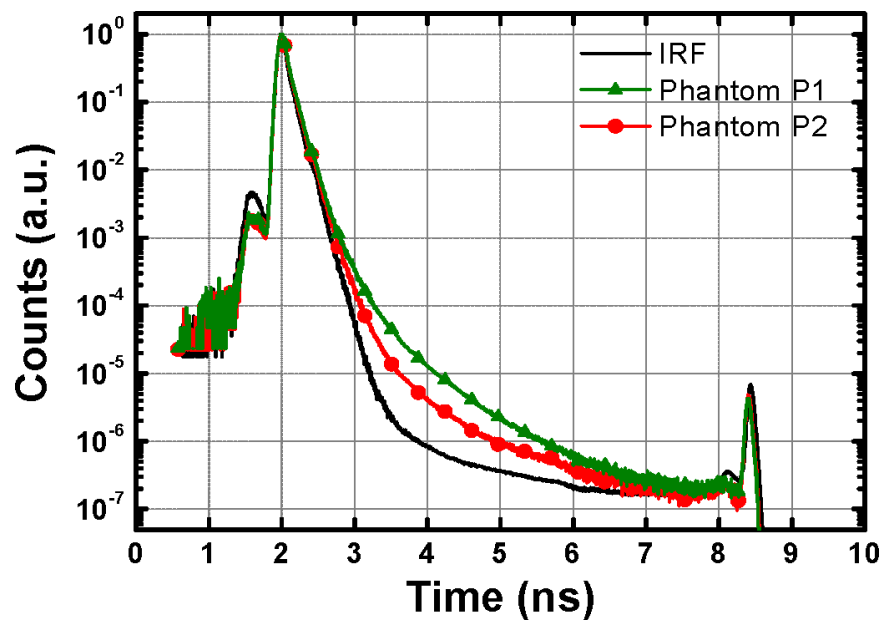

Fig. 10. Normalized instrument response function (solid line) and timeresolved reflectance curves corresponding to two homogeneous phantoms with different absorption coefficients.

spectroscopy with fast-gated SPAD equipment, thus improving the penetration depth capability even at small source-detector separation. However, for practical measurements it is not necessary to acquire the full reflectance curve, therefore the detector can be gated-on at the time delay corresponding to the mean penetration depth of interest, thus further strongly reducing the measurement time because only part of the reflectance curve is measured.

The reported dynamic range is limited to seven orders of magnitude because of very small reflections in optical fibers and connectors (see the small peak at the end of the curve in Fig. 9). By improving the optical path, it is possible to eliminate or at least reduce such reflections in order to employ higher excitation power, thanks to more powerful lasers, thus extending the dynamic range even further. In our setup, we always used a power lower than $2 \mathrm{~mW}$ even at the longer (6 ns) gate-ON delays. If a larger illumination area is chosen, the injected power could be further increased, yielding a corresponding improvement in dynamic range.

The maximum laser power injected into the phantom or the biological tissue is not limited by the maximum count rate that must be respected in TCSPC measurements before introducing significant distortions [10]. At longer gate-ON delays the power could be significantly increased in order to effectively detect the faint signal thus increasing the dynamic range. Also the TCSPC technique can offer a potentially unlimited dynamic range, but the measurement time would be too long and unpractical. Instead our setup allows a drastic reduction of the measurement time necessary to collect optical waveforms with the desired wide dynamic range.

The effectiveness of the proposed technique to increase penetration depth, spatial resolution, and contrast for noninvasive assessment of cerebral oxygenation has already been already demonstrated [11]. However, it can also be employed to increase performance in other applications such as time-resolved optical mammography [22], optical molecular imaging [23], and characterization of very low-density fluorophores. 
A possible limitation of our setup is the small active-area of the detector (100 $\mu \mathrm{m}$ diameter), but collection efficiency can be further increased by employing larger area SPADs (such as $200 \mu \mathrm{m}$ devices) or by using arrays of SPADs [24], [25].

\section{CONCLUSION}

In conclusion, we present a novel measurement technique able to drastically boost the dynamic range of time-resolved optical measurements of faint signals induced by much stronger laser pulse injection, at the same wavelength. In particular, we focused our technique to time-resolved near-infrared spectroscopy for photon-migration measurements.

The instrumental setup is based on an ultrafast time-gated SPAD. The main feature of the equipment is the rejection of early photons by quickly enabling the SPAD (in less than $500 \mathrm{ps}$ ) within a gate-ON time window, right after an adjustable timedelay from the laser injection. In this way, the laser power can be strongly increased to enhance the number of late photons to be detected in such a gate-on window, while avoiding statistical distortion of the single-photon counting techniques. We measured the IRF and the reflectance curves of two different homogeneous liquid phantoms at the minimum source-detector separation of $2 \mathrm{~mm}$. We obtained an extremely wide dynamic range of $10^{7}$ in a 33.5-min measurement time, which allowed the detection of photons delayed up to $6 \mathrm{~ns}$ from the laser peak with 95 ps time resolution. This result represents a reduction of three orders of magnitude in the measurement time needed to reach the same dynamic range with the standard free-running technique.

Moreover, we believe that the presented ultrafast time-gated detector technique could have great impact also on other biomedical applications, such as prompt detection of breast cancers or time-resolved optical molecular imaging.

\section{REFERENCES}

[1] A. Yodh and B. Chance, "Spectroscopy and imaging with diffusing light," Phys. Today, vol. 48, no. 3, pp. 34-40, Mar. 1995.

[2] Y. Hoshi and M. Tamura, "Detection of dynamic changes in cerebral oxygenation coupled to neuronal function during mental work in man," Neurosci. Lett., vol. 150, no. 1, pp. 5-8, Feb. 1993.

[3] D. J. Watmough, "Transillumination of breast tissues: factors governing optimal imaging of lesions," Radiology, vol. 147, pp. 89-92, Apr. 1983.

[4] R. Weissleder and U. Mahmood, "Molecular imaging," Radiology, vol. 219, no. 2, pp. 316-333, May 2001.

[5] J. Steinbrink, H. Wabnitz, H. Obrig, A. Villringer, and H. Rinneberg, "Determining changes in NIR absorption using a layered model of the human head," Phys. Med. Biol., vol. 46, no. 3, pp. 879-896, Mar. 2001.

[6] S. Del Bianco, F. Martelli, and G. Zaccanti, "Penetration depth of light re-emitted by a diffusive medium: Theoretical and experimental investigation," Phys. Med. Biol., vol. 47, no. 23, pp. 4131-4144, Dec. 2002.

[7] J. Selb, J. J. Stott, M. A. Franceschini, A. G. Sorensen, and D. A. Boas, "Improved sensitivity to cerebral hemodynamics during brain activation with a time-gated optical system: Analytical model and experimental validation," J. Biomed. Opt., vol. 10, no. 1, pp. 011013-1-011013-12, Jan. 2005.

[8] A. H. Hielscher, H. Liu, B. Chance, F. K. Tittel, and S. L. Jacques, "Timeresolved photon emission from layered turbid media," Appl. Opt., vol. 35, no. 4, pp. 719-728, Feb. 1996.

[9] A. Torricelli et al., "Time-resolved reflectance at null source-detector separation: Improving contrast and resolution in diffuse optical imaging," Phys. Rev. Lett., vol. 95, no. 7, pp. 078101-1-078101-4, 2005.

[10] W. Becker, Advanced Time-Correlated Single Photon Counting Techniques.. $\quad$ New York: Springer-Verlag, 2005, ISBN: 978-3-540-26047-9.
[11] A. Pifferi et al., "Time-resolved diffuse reflectance using small sourcedetector separation and fast single-photon gating," Phys. Rev. Lett., vol. 100, no. 13, pp. 138101-1-138101-4, Apr. 2008.

[12] R. H. Haitz, "Mechanisms contributing to the noise pulse rate of avalanche diodes," J. Appl. Phys., vol. 36, no. 10, pp. 3123-3131, Oct. 1965.

[13] S. Cova, M. Ghioni, A. Lacaita, C. Samori, and F. Zappa, "Avalanche photodiodes and quenching circuits for single-photon detection," Appl. Opt., vol. 35, no. 12, pp. 1956-1963, Apr. 1996.

[14] A. Gulinatti, P. Maccagnani, I. Rech, M. Ghioni, and S. Cova, "35 ps time resolution at room temperature with large area single photon avalanche diodes," Electron. Lett., vol. 41, no. 5, pp. 272-274, Mar. 2005.

[15] Micro Photon Devices S. r. 1. Detection Modules Data-sheets. (2009) [Online]. Available: www.microphotondevices.com

[16] A. Tosi, A. Dalla Mora, and F. Zappa, "All-silicon 1.55- $\mu$ m high-resolution photon counting and timing," IEEE Photon. Technol. Lett., vol. 20, no. 23, pp. 1956-1958, Dec. 2008.

[17] F. Zappa, M. Ghioni, S. Cova, C. Samori, and A. C. Giudice, "An integrated active-quenching circuit for single-photon avalanche diodes," IEEE Trans. Instrum. Meas., vol. 49, no. 6, pp. 1167-1175, Dec. 2000.

[18] F. Zappa, A. Tosi, and S. Cova, "InGaAs SPAD and electronics for low time jitter and low noise," in Proc. SPIE 6583, Photon Counting Appl., Quantum Opt., Quantum Cryptography, Prague, Czech Republic, 2007, pp. 65830E1-65830E12.

[19] D. S. Bethune, R. G. Devoe, C. Kurtsiefer, C. T. Retterner, and W. P. Risk, "System for gated detection of optical pulses containing a small number of photons using an avalanche photodiode," U.S. Patent 6218657, Apr. 17, 2001.

[20] A. Tomita and K. Nakamura, "Balanced gated-mode photon detector for quantum-bit discrimination at $1550 \mathrm{~nm}, "$ Opt. Lett., vol. 27, no. 20, pp. 1827-1829, Oct. 2002.

[21] L. Spinelli, F. Martelli, A. Farina, A. Pifferi, A. Torricelli, R. Cubeddu, and G. Zaccanti, "Calibration of scattering and absorption properties of a liquid diffusive medium at NIR wavelengths. Time-resolved method," Opt. Exp., vol. 15, no. 11, pp. 6589-6604, May 2007.

[22] P. Taroni, A. Torricelli, L. Spinelli, A. Pifferi, F. Arpaia, G. Danesini, and R. Cubeddu, "Time-resolved optical mammography between 637 and $985 \mathrm{~nm}$ : Clinical study on the detection and identification of breast lesions," Phys. Med. Biol., vol. 50, pp. 2469-2488, Nov. 2004.

[23] G. M. Turner, G. Zacharakis, A. Soubret, J. Ripoll, and V. Ntziachristos, "Complete-angle projection diffuse optical tomography by use of early photons," Opt. Lett., vol. 30, no. 4, pp. 409-411, Feb. 2005.

[24] F. Zappa, "Single-photon imaging at 20000 frame/s," Opt. Lett., vol. 30, no. 22, pp. 3024-3026, Nov. 2005

[25] S. Tisa, F. Guerrieri, A. Tosi, and F. Zappa, " 100 kframe/s 8 bit monolithic single-photon imagers," in Proc. 38th Eur. Solid-State Device Res. Conf. Edinburgh, U.K., 2008, pp. 274-277.

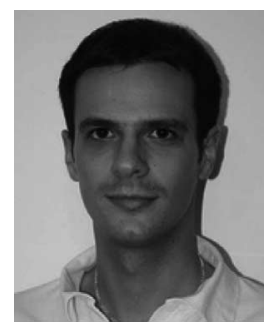

Alberto Dalla Mora was born in Fiorenzuola d'Arda, Italy, in 1981. He graduated summa cum laude in electronic engineering from the Politecnico di Milano, Milan, Italy, in 2006. He has been working toward the Ph.D. degree in information and communication technology in the Dipartimento di Elettronica e Informazione, Politecnico di Milano, since 2007.

His current research interests include the electronics development for visible and near-infrared single photon avalanche diodes (silicon, InGaAs, and germanium detectors) in fast gating regime and at highcount rate for applications in biomedical engineering and telecommunications.

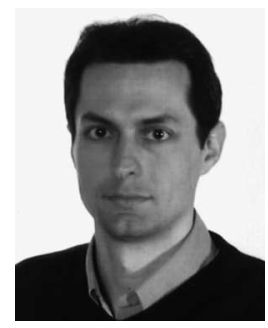

Alberto Tosi (M'07) was born in Borgomanero, Italy, in 1975 . He graduated summa cum laude in electronics engineering and received the Ph.D. degree in information and communication technology from the Politecnico di Milan, in 2001 and 2005, respectively.

Since 2006, he has been an Assistant Professor in the Dipartimento di Elettronica e Informazione, Politecnico di Milano. During 2004, he was a Summer Student with IBM T. J. Watson Research Center, Yorktown Heights, NY, where he was engaged in the experimental investigation and testing of very large scale integration complimentary metal-oxide-semiconductor circuits by means of single-photon detectors. His current research interests include visible and near-infrared single-photon detectors (silicon, germanium, and InGaAs detectors) and the related electronics for high-resolution timing applications. 


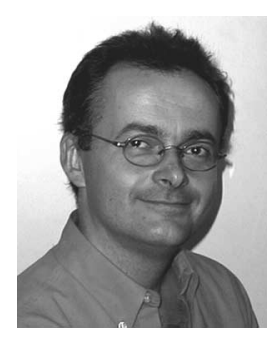

Franco Zappa (M'00-SM'07) was born in Milan, Italy, in 1965. He graduated in electronics engineering and received the Ph.D. degree in electronics from the Politecnico di Milano, Milan, in 1989 and 1993, respectively.

Since 1998, he has been an Associate Professor of electronics in the Dipartimento di Elettronica e Informazione, Politecnico di Milano. He is also with Istituto di Fotonica e Nanotecnologie-Consiglio Nazionale delle Ricerche (IFN-CNR), Milan, Italy. In 2005, he along with other colleagues established the university spin-off company Micro Photon Devices, S. r. 1., Bolzano. His current research interests include the design and applications of avalanche photodiodes in the visible and near-infrared wavelength ranges, and the design of photodetector arrays for imaging and the related electronics. He is the coauthor of three international patents in the field of integrated electronics and devices for single-photon detection. He is also the author or coauthor of about 80 technical papers.

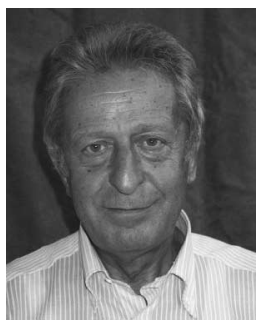

Sergio Cova (M'71-SM'82-F'92-LF'05) received the $\mathrm{Ph} . \mathrm{D}$. degree in nuclear engineering from the Politecnico di Milano, Milan, Italy, in 1962.

Since 1977, he has been a full-time Professor of electronics in the Dipartimento di Elettronica e Informazione and IIT Research Unit, Politecnico di Milano. During 2005, he along with other colleagues established the university spin-off company Micro Photon Devices S. r. L, Bolzano. He has been engaged in the research and development of detectors for optical and ionizing radiations, of microelectronic devices and circuits, of electronic and optoelectronic measurement instrumentation systems. In this frame, he carried out also interdisciplinary work in collaboration with researchers in physics, astronomy, biochemistry, and molecular biology. He pioneered the development of silicon single-photon avalanche diodes (SPAD) and the extension of photon counting techniques to the infrared spectral range with devices in germanium and III-V semiconductors. He invented the active-quenching circuit that opened the way to the application of SPADs and developed it up to monolithic integrated form. He is the author of more than 200 papers in international journals and conferences. He holds four U.S. and EU patents.

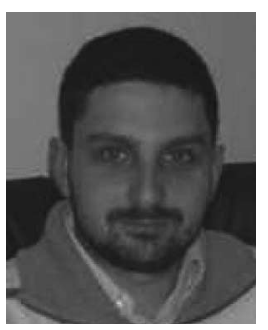

Davide Contini was born in Angera, Italy, in 1978 He received the Master's degree in electronics engineering from the Politecnico di Milano, Milan, Italy, in 2003, and the Ph.D. degree in physics from Dipartimento di Fisica, Politecnico di Milano, in 2007.

In 2004, he joined the Biomedical Optics Group in the Dipartimento di Fisica, Politecnico di Milano. From 2007 to 2008, he was a Postdoctoral Fellow with the Politecnico di Milano.

Since 2008, he has been an Assistant Professor in the Dipartimento di Fisica and ULTRAS-INFMCNR, Politecnico di Milano. His current research interests include the interaction of laser light with matter, and in particular the time-resolved spectroscopy of highly diffusive media for applications in biology and medicine.

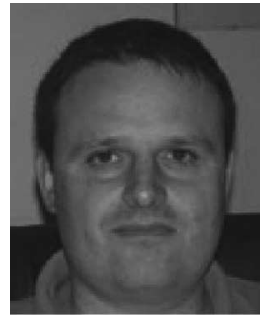

Antonio Pifferi was born in Milan, Italy, in $1965 . \mathrm{He}$ received the Master's degree in nuclear engineering, and the Ph.D. degree in physics from the Politecnico di Milano, Milan, in 1991 and 1995, respectively.

Since 1996, he has been in the Dipartimento di Fisica and IIT Research Unit, Politecnico di Milano, first as Researcher, and then as an Associate Professor in 2003. His current research interests include the development and application of new laser-based techniques and instrumentation for biomedical diagnosis, in particular, in the study of photon migration through highly scattering media with applications to optical tissue characterization, optical mammography, and tissue oximetry.

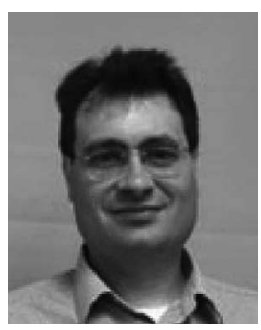

Lorenzo Spinelli received the M.S. and Ph.D. degrees in physics from the University of Milan, Milan, Italy, in 1994 and 1999, respectively.

Since 1999, he has been a Postdoctoral Fellow with the Dipartimento di Fisica, IFN-CNR Politecnico di Milano, Milan. In 2001, he became a Researcher for the Italian Research National Council, Politecnico of Milan. He was engaged in the research of structures developing in the section of broad area radiation beams. In particular, he focused his research to cavity solitons, which are stationary self-organized bright intensity peaks over a homogeneous background. His current research interest includes the study of photon migration in turbid media for optical biopsy and imaging.

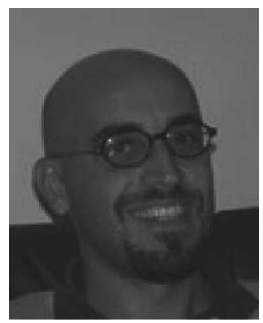

Alessandro Torricelli was born in Modena, Italy, in 1968. He received the Master's degree in electronics engineering from the Politecnico di Milano, Milan, Italy, in 1994, and the Ph.D. degree in physics from the Politecnico di Torino, Torino, Italy, in 1999.

In 1995, he joined for one year the "Mario Negri” Institute for Pharmacological Research, Milan, where he was engaged on flow cytometry and cell kinetics. Since 1993, he has been with the Dipartimento di Fisica and IIT Research Unit, Politecnico di Milano, where his research interests include timeresolved diffuse spectroscopy and optical imaging. Since 2005, he has been an Associate Professor in physics with the Politecnico di Milano.

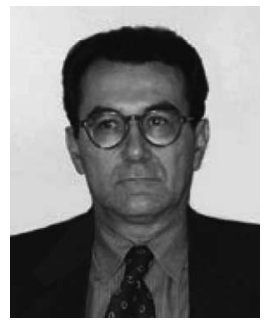

Rinaldo Cubeddu was born in Alessandria, Italy, on July 12,1944 . He received the Ph.D. degree in physics from the University of Pavia, Pavia, Italy, in 1967.

He joined the Dipartimento di Fisica, IIT Research Unit, IFN-CNR, Politecnico di Milano, where he became Assistant Professor in optics in 1983 and a fulltime Professor in physics, in 1986. He is currently the Head of the Department of Physics, Politecnico di Milano.

His research interests include lasers and their applications to biology and medicine. He has developed instrumentation for time-resolved fluorescence spectroscopy and imaging for application to photodynamic therapy and diagnosis. More recently, he has been involved in the field of photon migration for applications in mammography, oxymetry, and functional imaging. Besides the field of biomedicine, the instrumentation developed has been applied for diagnostic purposes in agriculture for the evaluation of internal fruit quality and in cultural heritage for the fluorescence analysis of fresco paintings and marble statues. 